\title{
Evaluation of Real-Time 16S rDNA PCR and Pyrosequencing for Routine Identification of Bacteria in Joint Fluid and Tissue Specimens
}

\author{
Naomi J. Gadsby ${ }^{1}$, Alev Onen ${ }^{1}$, Sally-Anne Phillips ${ }^{2}$, Luke Tysall ${ }^{1}$, Steffen J. Breusch ${ }^{2}$, \\ Hamish Simpson ${ }^{2}$, Jayshree Dave ${ }^{1,3}$, Elzbieta Czarniak ${ }^{1}$, Kate E. Templeton ${ }^{1}$ \\ ${ }^{1}$ Microbiology, Department of Laboratory Medicine, Royal Infirmary of Edinburgh, Edinburgh, United Kingdom \\ ${ }^{2}$ Department Orthopaedic Surgery, Royal Infirmary of Edinburgh, Edinburgh, United Kingdom \\ ${ }^{3}$ Medical Microbiology, St. George's Hospital, London, United Kingdom \\ E-mail:naomi.gadsby@luht.scot.nhs.uk
}

Received November 4, 2011; revised November 11, 2011; accepted December 5, 2011

\begin{abstract}
16S rDNA PCR and sequencing are powerful tools for bacterial detection and identification, although their routine use is not currently widespread in the field of clinical microbiology. The availability of pyrosequencing now makes $16 \mathrm{~S}$ rDNA assays more accessible to routine diagnostic laboratories, but this approach has had limited evaluation in general diagnostic practice. In this study we evaluated a real-time 16S rDNA PCR and pyrosequencing assay for use in a routine microbiology laboratory, by retrospectively testing joint fluid and joint tissue specimens received for conventional culture. We found that use of the real-time $16 \mathrm{~S}$ rDNA assay was clinically valuable in this specimen type because it enabled us to identify a small number of culture-negative infections. Although faster and less labour-intensive, we found that the utility of pyrosequencing for pathogen identification is still hampered by shorter read lengths compared to conventional (Sanger) sequencing. Combining results from both molecular and conventional culture methods, bacteria were only detected in $11.8 \%$ specimens in this study. However, the detection rate was increased to $18.6 \%$ if specimens were only included from patients with a documented clinical suspicion of infection. In conclusion, while pyrosequencing had significant advantages in speed and ease-of-use over conventional sequencing, multiple reactions will be required to deliver comparable species-level identification, thus negating many of the benefits of using the technique. We found that $16 \mathrm{~S}$ rDNA PCR and sequencing should be rationally targeted on the basis of good clinical information in the routine diagnostic setting, and not used as a general screening test for the exclusion of bacterial infection in joint specimens.
\end{abstract}

Keywords: 16S rRNA, Real-Time PCR, Sequencing, Pyrosequencing, Orthopaedic Infection

\section{Introduction}

Diagnosis of bacterial infection is routinely made through the microbiological culture of clinical specimens. However, despite overt clinical signs of infection, these specimens may be falsely negative due to the presence of nutritionally fastidious organisms or the prior use of antibiotics. Without successful bacterial isolation, patients may continue to be treated empirically with broad-spectrum antibiotics. Therefore, the development of improved bacterial diagnostic assays is integral to efforts to improve antibiotic stewardship.

Amplification and sequencing of regions of the pan- prokaryotic 16S rRNA gene has been helpful in identifying culture-negative bacterial infections in a number of studies [1-3]. However, conventional PCR, gel-based detection and sequencing methodologies mean that routine testing is labour-intensive and requires considerable financial investment and technical expertise [4]. A number of large clinical microbiology laboratories have made this investment, and may also offer testing as a reference service to other laboratories. However, the ability to perform such testing in-house in the local diagnostic laboratory would be of significant benefit in reducing costs and turn-around-times.

Real-time 16S rDNA PCR followed by pyrosequencing 
would be a quicker and simpler alternative for smaller routine diagnostic laboratories. Pyrosequencing is a method of sequencing by synthesis, the principles of which have been reviewed elsewhere [5]. Pyrosequencing requires significantly less hands-on time, with fewer steps and less complexity compared to the conventional (Sanger) sequencing process, however, pyrosequencing read lengths are limited compared to those of over 500 nucleotides achieved by the conventional method. Despite this, pyrosequencing has recently been applied in $16 \mathrm{~S}$ rDNA PCR studies; for example, differentiation of bacteria into either Gram-positive or Gram-negative groups was possible using only 3 nucleotides of pyrosequence data [6] and bacteria were identified to at least the genus level using $20-30$ nucleotides from more than one locus in the $16 \mathrm{~S}$ and/or $23 \mathrm{~S}$ rRNA genes [7-9]. However, as sequence read lengths have improved over time to around 50 - 80 nucleotides, pyrosequencing may now be a more feasible option for identification to the species level [10].

Pyrosequencing has a number of current applications in clinical microbiology in addition to bacterial identification using 16S rDNA PCR, including single-nucleotide polymorphism-based detection of drug resistance in $M y$ cobacterium tuberculosis [11] and Influenza A [12]. Pyrosequencing technology is also becoming more accessible for routine diagnostic Microbiology laboratories, particularly due to increased sharing of resources across other clinical laboratory disciplines such as Virology and Molecular Pathology.

The objective of this study was to assess the value of using a real-time $16 \mathrm{~S}$ rDNA PCR and sequencing approach as a general screening test for the joint fluid and tissue specimens which we routinely receive in our laboratory, and which are frequently culture-negative. We retrospectively tested joint fluid and tissue specimens from 100 patients and compared the performance of a real-time $16 \mathrm{~S}$ rDNA assay incorporating either conventional sequencing or pyrosequencing methodology, with routine bacteriological culture at the time of specimen submission.

\section{Methods}

\subsection{Clinical Specimens}

Study specimens were not subjected to any selection based on clinical criteria, in order to form a representative collection of those routinely received by the laboratory. 152 specimens from 100 patients, submitted to the Microbiology Laboratory at the Royal Infirmary of Edinburgh between February and May 2010 for routine culture, were available for retrospective testing by PCR. The specimen collection comprised 66 (43.4\%) surgical joint tissues and $86(56.6 \%)$ joint fluid aspirates, with 64 speci- mens (42.1\%) from native joints and 88 (57.9\%) specimens from joints containing prosthetic material. At the time of sampling, 9/100 patients were undergoing antibiotic treatment, 76/100 patients were not undergoing antibiotic treatment and this information was not available for $15 / 100$ patients.

Routine culture comprised 48 hour $37^{\circ} \mathrm{C}$ incubation on blood agar, chocolate blood agar and anaerobic blood agar plates, and in Schaedler broth, followed by biochemical identification of isolates. As standard operating procedure, specimens were processed for culture and then promptly stored at $4^{\circ} \mathrm{C}$ until the normal point of discard, between 1 and 4 weeks later. At this point, specimens were transferred to $-70^{\circ} \mathrm{C}$ storage for the study and linked to available clinical data (full data was available for $87 / 100$ patients). Handling and testing of specimens for the study was carried out in accordance with local ethical approval (South East Scotland SAHSC Human Annotated BioResource reference No.10/S1402/33).

\subsection{Real-Time 16S rDNA PCR}

DNA was extracted from stored specimens by DNeasy Blood and Tissue kit (Qiagen) according to the manufacturer's protocols for tissues and Gram-positive bacteria (including proteinase $\mathrm{K}$ and lysozyme digestion). Extracts were diluted 1:10 to reduce PCR inhibition. A realtime $16 \mathrm{~S}$ rDNA PCR assay was used to amplify a $567 \mathrm{bp}$ region at the 5' end of the 16S rRNA gene as previously described [13]. One negative extraction control was added for every batch of 11 - 15 samples processed and a negative PCR run control was included for every 10 extracts tested. 5 inhibited specimens were considered as PCR negative for the purposes of assay evaluation. As has been well described $[2,13,14]$, negative controls for both extraction and PCR processes were positive using 16S rDNA PCR; Ct values ranged from 34 to 36 . Specimens with $\mathrm{Ct}$ values $\leq 34$ were sequenced to determine positivity.

\subsection{Sequencing}

Conventional (Sanger) sequencing was carried out using ABI Prism BigDye Terminator and the ABI 3730 instrument (Applied Biosystems). Pyrosequencing was carried out using the Pyromark Q24 vacuum workstation and PyroMark Q24 instrument (Qiagen) with a dispensation order of 20(CTGA). The PCR method was adapted for pyrosequencing by use of a $5^{\prime}$ ' biotinylated forward PCR primer and a pyrosequencing primer with the same oligonucleotide sequence as the PCR probe [13]. Sequence reads were used to query the nucleotide collection of the GenBank database using the nucleotide BLAST program with search criteria for highly similar sequences and exclusion 
of uncultured or environmental isolates. Conventional sequence read lengths ranged from 483 - $563 \mathrm{bp}$, with $\geq 99 \%$ coverage and $\geq 99 \%$ identity used as the criteria for species assignment. Positive specimens gave strong signal intensity and clear traces; these were distinct in appearance from the very mixed traces of low signal intensity given by negatives. Positives were also pyrosequenced for comparison of the ability to identify the bacteria present. Pyrosequence read lengths ranged from 22 - $46 \mathrm{bp}$, which was sufficient in most cases to assign a genus but not species level identification, based on $100 \%$ coverage and $100 \%$ identity with other sequences in the GenBank database.

\section{Results}

\subsection{Comparison of 16S rDNA Assay and Culture}

Of the 152 specimens in this specimen collection, a total of $18(11.8 \%)$ gave positive results; 12 were positive by both culture and 16S rDNA assay, 3 were positive by $16 \mathrm{~S}$ rDNA assay only and 3 were positive by culture only (Table 1). Compared to culture, the sensitivity of the $16 \mathrm{~S}$ rDNA assay was $80.0 \%$ and the specificity was $97.8 \%$. Overall positivity rates for the individual methods were the same: $15 / 152(9.9 \%)$ specimens positive by $16 \mathrm{~S}$ rDNA assay and $15 / 152(9.9 \%)$ by routine culture.
Three culture-negative specimens were positive by 16S rDNA assay (Table 2). The clinical relevance of the additional positive specimens was apparent in two cases. The first case was Streptococcus agalactiae detected in an aspirate from a prosthetic hip joint; the patient was being treated with intravenous penicillin for Group B streptococcal septicaemia with suspected haematogenous spread to the joint. The second case was Haemophilus influenzae detected in an aspirate from a native hip joint in a 3-year-old child; the patient had suspected septic arthritis and was treated empirically. In an additional case, Staphylococcus aureus was detected in a femoral tissue specimen from a patient with a prosthetic hip; interpretation of this result was difficult because a duplicate tissue

Table 1. Concordance of retrospective testing by real-time $16 S$ rDNA assay with routine microbiological culture at the time of specimen submission.

\begin{tabular}{lccc}
\hline & & \multicolumn{2}{c}{ Bacterial culture } \\
\cline { 3 - 4 } & & Positive & Negative \\
\hline \multirow{2}{*}{ 16S rDNA assay } & Positive & $12^{*}$ & 3 \\
& Negative & $3^{\#}$ & $134^{\S}$ \\
\hline
\end{tabular}

*Two were identified as mixed infections by culture. ${ }^{*}$ Includes 2 inhibited PCR reactions. ${ }^{\S}$ Includes 3 inhibited PCR reactions.

Table 2. Pathogen identification in specimens from 13 patients by routine culture and biochemical methods compared to real-time 16S rDNA PCR and conventional sequencing or pyrosequencing.

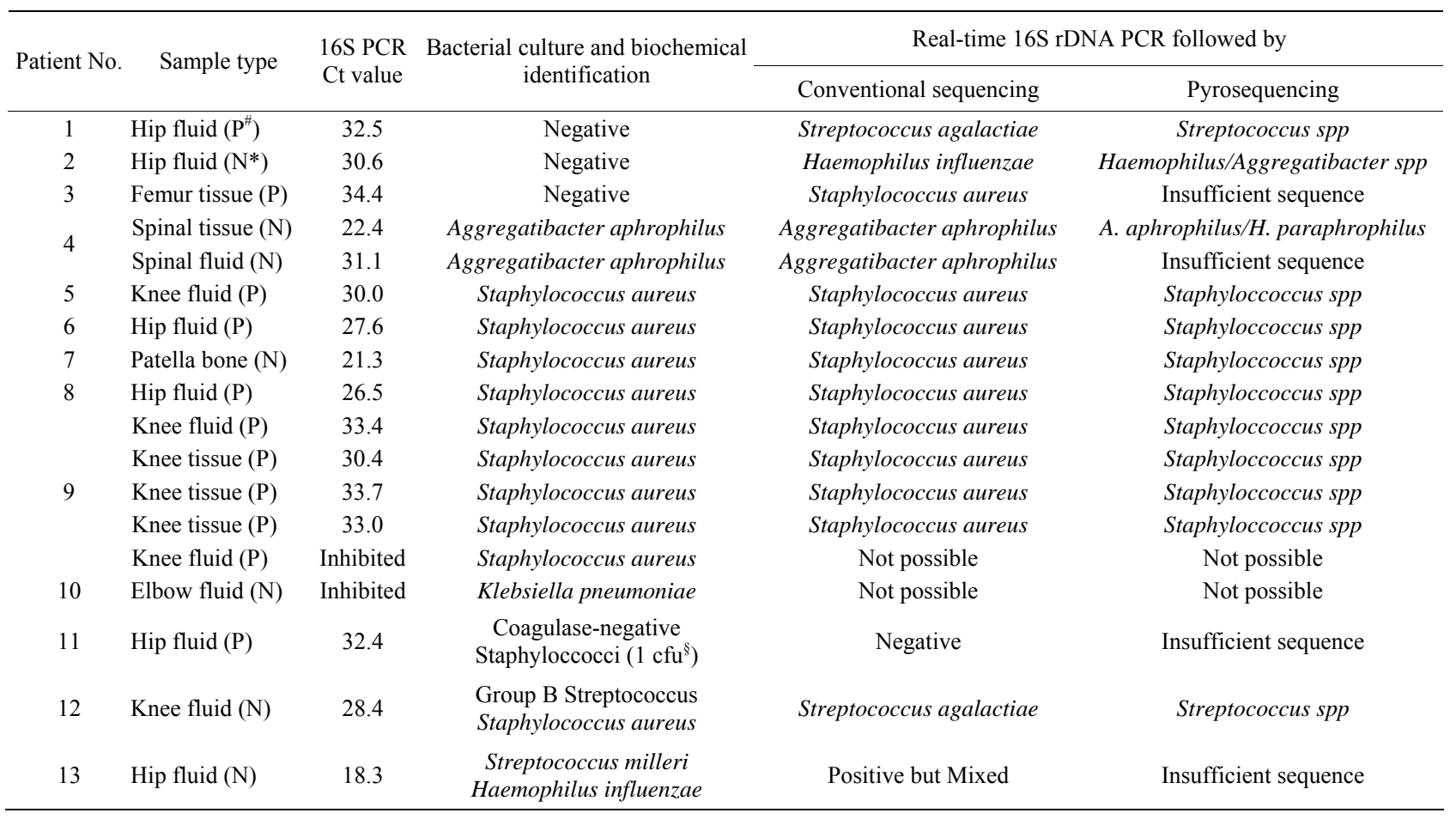

${ }^{\#}$ Specimen from joint containing prosthetic material. ${ }^{*}$ Specimen from native bone/joint. ${ }^{\S}$ Colony forming unit. 
specimen was negative by both $16 \mathrm{~S}$ rDNA assay and culture, and S. aureus is part of normal skin flora as well as a true pathogen.

Three culture-positive specimens were negative by $16 \mathrm{~S}$ rDNA assay; the PCR was inhibited in two cases and a low bacterial load of doubtful clinical significance (1 colony forming unit (cfu) coagulase-negative Staphylococcus) was found in the remaining case (Table 2). There were two mixed infections identified by culture; in one case a mixed sequence trace was generated but the PCR was strongly positive, and in the other, the trace was not mixed, presumably demonstrating amplification of the dominant organism only.

\subsection{Comparison of Conventional Sequencing and Pyrosequencing}

A genus-level identification using pyrosequencing was obtained for 12 of the $14(85.7 \%)$ specimens identified to the species level by conventional sequencing (Table 2). In the remaining 2 cases, sequence reads were not long enough to enable a specific genus-level match in the GenBank database.

\subsection{Analysis of Clinical Data}

Despite combining PCR and culture results, overall in this study bacteria were detected in only 18/152 (11.8\%) specimens from $13 / 100$ patients (13.0\%). Therefore, as full clinical data were available for $87 / 100$ patients, records were examined for evidence of clinical suspicion of infection (CSI) based on recorded signs of sepsis, erythema, swelling, pain, loss of function, loosening of prosthesis/ joint, raised inflammatory markers, or previous evidence of infection. We found that CSI was noted in only $49 / 87$ $(56.3 \%)$ patients from whom specimens were sent for culture; 23 had suspected native bone/joint infection and 26 had suspected prosthetic joint infection. Of the 49 patients with CSI, $11(22.4 \%)$ had positive specimens, including three only identified by the $16 \mathrm{~S}$ rDNA assay. In contrast, of the 38 patients without CSI, none had positive specimens. Of the 13 patients for whom insufficient clinical information was available, two had positive specimens. Therefore, more rational targeting of specimens from patients with CSI would have substantially increased the detection rate from $13 \%$ to $22.4 \%$ of patients and from $11.8 \%$ to $18.6 \%$ of specimens.

\section{Conclusions}

16S rDNA PCR and sequencing holds much promise as a molecular tool for the diagnosis of culture negative infections, enabling us to reduce broad-spectrum antibiotic use. However, the challenge is to integrate it into widespread use in the routine microbiology laboratory. Although 16S rDNA PCR and sequencing are already in use in some clinical microbiology laboratories, this requires considerable specialist equipment and expertise. The availability of pyrosequencing has the potential to make 16S rDNA assays more accessible to routine diagnostic laboratories as it offers a significantly less labourand resource-intensive approach, however, several issues remain to be resolved.

In this study, although pyrosequencing was rapid to perform, and the ease of use of the small PyroMark Q24 instrument made it well-suited to a routine laboratory, read lengths achieved by de novo sequencing were insufficient for species discrimination. Pyrosequencing has been found to generate suitable traces for identification with bacterial loads $\geq 10^{4} \mathrm{cfu} / \mathrm{ml}[10]$, and this was consistent with our own findings (data not shown). With either sequencing method, we found that assigning an identity from 16S rDNA sequence reads using GenBank was not straight-forward. Due to a lack of phylogenetic curation of the database, some sequence entries appear to be incorrectly assigned to particular species. As we found in this study, pyrosequencing of a single region within the 16S rRNA gene using the small Pyromark Q24 instrument can still not deliver the identification to the species level which can be achieved by conventional sequencing. Combining pyrosequence data from more than one fragment of the 16S rRNA gene has been more successful at differentiation $[7,8]$, but a combination of sequences from hypervariable regions 2,3 and 6 appears to be optimal [15]. This requires multiple reactions and more sequence analysis, which is less convenient for a routine laboratory.

Sequence quality of entries in the GenBank database is uncertain, with many apparently mis-identified. This means that widespread use in the routine laboratory would require the purchase or in-house construction of a standardised, clinically relevant sequence database [10] in order to achieve acceptable levels of quality and ease of use. A further issue is the generation of mixed sequence traces from specimens with more than one bacterial species present. Although polybacterial infections can now be significantly differentiated using a combination of different $16 \mathrm{~S}$ rDNA PCRs and commercial software [16], this is an expensive approach for low sample numbers. However, in a recent study, pyrosequencing was able to accurately identify both organisms in $5 / 16$ specimens with dual infection, with the use of an in-house database [10].

The use of $16 \mathrm{~S}$ rDNA PCR is not a perfect pan-bacterial diagnostic strategy because it is not an optimal target for the differentiation of some bacterial species [15]. In addition, the sensitivity is reduced by the problem of amplification of background DNA contamination, as seen in the present study. The sensitivity of real-time $16 \mathrm{~S}$ rDNA 
PCR and sequencing compares relatively unfavourably to pathogen-specific real-time PCR, being at least 10 -fold less sensitive [13]. Therefore, specimens with low bacterial loads will be missed by using this technique; this may be a particular problem in specimens from prosthetic joint infections where bacteria are likely to be present largely in biofilms. However, as illustrated in this study, it is very useful in identifying bacteria present at higher levels but which are unlikely to grow on conventional culture media due to their fastidious nature or prior antibiotic treatment. Alternative molecular methods, such as performing a battery of species-specific PCR reactions on $16 \mathrm{~S}$ rDNA assay positive specimens [17], may circumvent the need for sequencing, but only enable detection of the limited number of organisms actively sought.

In this study, a 16S rDNA PCR assay was used to retrospectively test 152 joint fluid and tissue specimens for comparison to routine microbiological culture. We found good concordance between conventional culture methods and $16 \mathrm{~S}$ rDNA PCR, and were able to identify a small number of clinically significant additional positive results. There were no potential false positives using $16 \mathrm{~S}$ rDNA PCR and sequencing since all identifications were concordant with culture, and clinically relevant pathogens were detected in the three PCR positive cases which were culture-negative. However, overall positivity rates were unexpectedly low, therefore a full evaluation of the effectiveness of the 16S rDNA PCR assay compared to conventional culture in joint fluid and tissue specimens will require a larger study. In this study, combining results from both molecular and conventional culture methods, bacteria were only detected in 18/152 (11.8\%) specimens. Other similar studies have described composite culture and 16S rDNA PCR detection rates ranging from $18.5 \%$ - $62.5 \%$ in joint specimens $[1,2,17-19]$. Further investigation revealed a significant proportion of specimens in this study $(56 \%)$ were sent from patients without a documented suspicion of infection. Therefore, testing a subset of the received specimens, based on good clinical information, would have increased the detection rate from $11.8 \%$ to $18.6 \%$ of specimens and from $13 \%$ to $22.4 \%$ of patients. As even a single additional positive result is important for the management of the individual patient, this molecular assay would be a beneficial addition to routine culture, but only where specific clinical criteria are met; opportunistic sampling to exclude bacterial infection should be discouraged.

In conclusion, while pyrosequencing had significant advantages in speed and ease-of-use over conventional sequencing, multiple reactions are required to deliver comparable species-level identification, thus negating many of the benefits of using the technique. Furthermore, $16 \mathrm{~S}$ rDNA PCR should be rationally targeted on the basis of good clinical information in the routine diagnostic setting, and not used as a general screening test for the exclusion of bacterial infection in joint specimens.

\section{Acknowledgements}

The authors thank Mr Andrew Mitchell for assistance in obtaining data from the laboratory results database and the staff of the Microbiology Laboratory, Royal Infirmary of Edinburgh. This work was supported by NHS Education for Scotland (NES) through the Clinical Scientist Training Programme. Part of this study was presented in poster format at the 21st European Congress of Clinical Microbiology and Infectious Diseases on 9th May 2011. The authors declare no conflicts of interest.

\section{References}

[1] F. Fenollar, V. Roux, A. Stein, M. Drancourt and D. Raoult, "Analysis of 525 Samples to Determine the Usefulness of PCR Amplification and Sequencing of the 16S rRNA Gene for Diagnosis of Bone and Joint Infections," Journal of Clinical Microbiology, Vol. 44, No. 3, 2006, pp. 1018-1028. doi:10.1128/JCM.44.3.1018-1028.2006

[2] A. L. Rosey, E. Abachin, G. Quesnes, C. Cadilhac, Z. Pejin, C. Glorion, P. Berche and A Ferroni, "Development of a Broad-Range 16S rDNA Real-Time PCR for the Diagnosis of Septic Arthritis in Children," Journal of Microbiological Methods, Vol. 68, No. 1, 2007, pp. 88-93. doi:10.1016/j.mimet.2006.06.010

[3] P. E. Fournier, F. Thuny, H. Richet, H. Lepidi, J. P. Casalta, J. P. Arzouni, M. Maurin, M. Célard, J. L. Mainardi, T. Caus, F. Collart, G. Habib and D. Raoult, "Comprehensive Diagnostic Strategy for Blood Culture-Negative Endocarditis: A Prospective Study of 819 New Cases," Clinical Infectious Diseases, Vol. 51, No. 2, 2010, pp. 131140. doi: $10.1086 / 653675$

[4] K. A. Harris and J. C. Hartley, "Development of BroadRange 16S rDNA PCR for Use in the Routine Diagnostic Clinical Microbiology Service," Journal of Medical Microbiology, Vol. 52, No. 8, 2003, pp. 685-691. doi:10.1099/jmm.0.05213-0

[5] A. Ahmadian, M. Ehn and S. Hober, "Pyrosequencing: History, Biochemistry and Future," Clinica Chimica Acta, Vol. 363, No. 1-2, 2006, pp. 83-94. doi:10.1016/i.ccen.2005.04.038

[6] N. Kobayashi, T. W. Bauer, M. J. Tuohy, I. H. Lieberman, V. Krebs, D. Togawa, T. Fujishiro and G. W. Procop, "The Comparison of Pyrosequencing Molecular Gram Stain, Culture and Conventional Gram Stain for Diagnosing Orthopaedic Infections," Journal of Orthopaedic Research, Vol. 24, No. 8, 2006, pp. 1641-1649. doi:10.1002/jor.20202

[7] J. A. Jordan, A. R. Butchko and M. B. Durso, "Use of Pyrosequencing of $16 \mathrm{~S}$ rRNA Fragments to Differentiate between Bacteria Responsible for Neonatal Sepsis," The 
Journal of Molecular Diagnostics, Vol. 7, No. 1, 2005, pp. 105-110. doi:10.1016/S1525-1578(10)60015-3

[8] R. A. Luna, L. R. Fasciano, S. C. Jones, B. L. Boyanton, T. T. Ton and J. Versalovic, "DNA PyrosequencingBased Bacterial Pathogen Identification in a Pediatric Hospital Setting," Journal of Clinical Microbiology, Vol. 45, No. 9, 2007, pp. 2985-2992. doi:10.1128/JCM.00630-07

[9] J. A. Jordan, J. Jones-Laughner and M. B. Durso, "Utility of Pyrosequencing in Identifying Bacteria Directly from Positive Blood Culture Bottles," Journal of Clinical Microbiology, Vol. 47, No. 2, 2009, pp. 368-372. doi:10.1128/JCM.01991-08

[10] J. Lu, R. Yu, Y. Yan, J. Zhang and X. Ren, "Use of Pyromark Q96 ID Pyrosequencing System in Identifying Bacterial Pathogen Directly with Urine Specimens for Diagnosis of Urinary Tract Infections," Journal of Microbiological Methods, Vol. 86, No. 1, 2011, pp. 78-81. doi:10.1016/j.mimet.2011.03.016

[11] C. Arnold, L. Westland, G. Mowat, A. Underwood, J. Magee and S. Gharbia, "Single-Nucleotide PolymorphismBased Differentiation and Drug Resistance Detection in Mycobacterium Tuberculosis from Isolates or Directly from Sputum," Clinical Microbiology and Infection, Vol. 11, No. 2, 2005, pp. 122-130. doi:10.1111/j.1469-0691.2004.01034.x

[12] R. A. Bright, D. K. Shay, B. Shu, N. J. Cox and A. I. Klimov, "Adamantane Resistance among Influenza A Viruses Isolated Early during the 2005-2006 Influenza Season in the United States," Journal of the American Medical Association, Vol. 295, No. 8, 2006, pp. 891-894. doi:10.1001/jama.295.8.joc60020

[13] C. Schabereiter-Gurtner, M. Nehr, P. Apfalter, A. Makristathis, M. L. Rotter and A. M. Hirschl, "Evaluation of a Protocol for Molecular Broad-Range Diagnosis of Culture-Negative Bacterial Infections in Clinical Routine Diagnosis," Journal of Applied Microbiology, Vol. 104,
No. 4, 2008, pp.1228-1237. doi:10.1111/j.1365-2672.2007.03648.x

[14] C. E. Corless, M. Guiver, R. Borrow, V. Edwards-Jones, E. B. Kaczmarski and A. J. Fox, "Contamination and Sensitivity Issues with a Real-Time Universal 16S rRNA PCR," Journal of Clinical Microbiology, Vol. 38, No. 5, 2000, pp. 1747-1752.

[15] S. Chakravorty, D. Helb, M. Burday, N. Connell and D. Alland, "A Detailed Analysis of 16S Ribosomal RNA Gene Segments for the Diagnosis of Pathogenic Bacteria," Journal of Microbiological Methods, Vol. 69, No. 2, 2007, pp. 330-339. doi:10.1016/j.mimet.2007.02.005

[16] O. Kommedal, K. Lekang, N. Langeland and H. G. Wiker, "Characterization of Polybacterial Clinical Samples Using a Set of Group-Specific Broad-Range Primers Targeting the 16S rRNA Gene Followed by DNA Sequencing and RipSeq Analysis," Journal of Medical Microbiology, Vol. 60, No. 7, 2011, pp. 927-936. doi:10.1099/jmm.0.028373-0

[17] S. Yang, P. Ramachandran, A. Hardick, Y. H. Hsieh, C. Quianzon, M. Kuroki, J. Hardick, A. Kecojevic, A. Abeygunawardena, J. Zenilman, J. Melendez, V. Doshi, C. Gaydos and R. E. Rothman, "Rapid PCR-Based Diagnosis of Septic Arthritis by Early Gram-Type Classification and Pathogen Identification," Journal of Clinical Microbiology, Vol. 46, No. 4, 2008, pp. 1386-1390. doi:10.1128/JCM.02305-07

[18] J. Jalava, M. Skurnik, A. Toivanen, P. Toivanen and E. Eerola, "Bacterial PCR in the Diagnosis of Joint Infection," Annals of the Rheumatic Diseases, Vol. 60, No. 3, 2001, pp. 287-289. doi:10.1136/ard.60.3.287

[19] H. Bonilla, R. Kepley, J. Pawlak, B. Belian, A. Raynor and L. D. Saravolatz, "Rapid Diagnosis of Septic Arthritis Using 16S rDNA PCR: A Comparison of 3 Methods," Diagnostic Microbiology \& Infectious Disease, Vol. 69, No. 4, 2011, pp. 390-395.

doi:10.1016/j.diagmicrobio.2010.11.010 